\title{
Paradigm Shift in Intra-Arterial Mechanical Thrombectomy for Acute Ischemic Stroke : A Review of Randomized Controlled Trials after 2015
}

\author{
Jae Jon Sheen, Young Woo Kim \\ Department of Neurosurgery, Uijeongbu St. Mary's Hospital, College of Medicine, The Catholic University of Korea, Uijeongbu, Korea
}

Three randomized control trials (RCTs), published in 2013, investigated efficacy of mechanical thrombectomy in large vessel occlusions and did not show better results compared to intravenous (IV) recombinant tissue-type plasminogen activator (tPA) alone. However, most clinicians treating stroke consider mechanical thrombectomy as the standard treatment rather than using IV tPA alone. This paradigm shift was based on five RCTs investigating efficacy of mechanical thrombectomy in acute ischemic stroke conducted from 2010 to 2015. They demonstrated that mechanical thrombectomy was effective and safe in acute ischemic stroke with anterior circulation occlusion when performed within 6 hours of stroke onset. There are four reasons underlying the different results observed between the trials conducted in 2013 and 2015. First, the three RCTs of 2013 used low-efficiency thrombectomy devices. Second, the three RCTs used insufficient image selection criteria. Third, following the initial presentation at the hospital, reperfusion treatment required a long time. Fourth, the three RCTs showed a low rate of successful recanalization. Time is the most important factor in the treatment of acute ischemic stroke. However, current trends utilize advanced imaging techniques, such as diffusion-weighted imaging and multi-channel computer tomographic perfusion, to facilitate the detection of core infarction, penumbra, and collateral flows. These efforts demonstrate that patient selection may overcome the barriers of time in specific cases.

Key Words : Stroke · Infarction · Thrombectomy.

\section{INTRODUCTION}

Three randomized control trials (RCTs) published in 2013 on mechanical thrombectomy for large vessel occlusions did not show better results compared to intravenous (IV) recombinant tissue-type plasminogen activator (tPA) alone. The results of the "Intra-arterial Versus Systemic Thrombolysis for
Acute Ischemic Stroke” (SYNTHESIS Expansion) indicated that mechanical thrombectomy was not superior to regular management using IV $\mathrm{tPA}^{6)}$. The "Interventional Management of Stroke Trial III" (IMS III) trial demonstrated no significant differences between the functional performance of patients undergoing mechanical thrombectomy and IV tPA, with similar safety outcomes"). Finally, "Mechanical Retrieval

- Received : June 26, 2019 •Revised : August 26, 2019 •Accepted : September 23, 2019

- Address for reprints : Young Woo Kim

Department of Neurosurgery, Uijeongbu St. Mary's Hospital, College of Medicine, The Catholic University of Korea, 271 Cheonbo-ro, Uijeongbu 11765, Korea Tel : +82-31-820-3619, E-mail : youngwookim1732@gmail.com, ORCID : https://orcid.org/0000-0002-9825-4927

This is an Open Access article distributed under the terms of the Creative Commons Attribution Non-Commercial License (http://creativecommons.org/licenses/by-nc/4.0) which permits unrestricted non-commercial use, distribution, and reproduction in any medium, provided the original work is properly cited. 
and Recanalization of Stroke Clots Using Embolectomy” (MR RESCUE) investigators showed that endovascular treatment of penumbra in patients was not as efficient as that of standard treatment with medications ${ }^{10)}$. These studies showed an unfavorable outcome with mechanical thrombectomy, causing reluctance in many clinicians to perform mechanical thrombectomy until 2015. However, recently, most stroke clinicians consider mechanical thrombectomy as the standard treatment rather than management with IV t-PA alone. We aimed to demonstrate this paradigm shift occurring after the five RCTs were published in 2015 2,5,7,9,14). Additionally, we introduced recent studies that helped clinicians overcome the long existing barrier of time.

\section{ADVANCEMENT OF MECHANICAL THROMBEC- TOMY}

Five RCTs investigating mechanical thrombectomy for acute ischemic stroke were carried out from 2010 to 2015.

The "Multicenter RCT of Endovascular Treatment for Acute Ischemic Stroke" (MR CLEAN) reported the results of 500 patients enrolled at 16 medical centers in the Netherlands ${ }^{2}$. The investigators used retrievable stents in 190 of the 233 patients $(81.5 \%)$ subjected to intra-arterial treatment. There was a significant difference of $13.5 \%$ in the rate of modified Rankin score (mRS 0 to 2 ) in favor of the mechanical thrombectomy, demonstrating that mechanical thrombectomy for acute ischemic stroke associated with anterior circulation occlusion was effective and safe, if performed within 6 hours of stroke onset.

In the "Endovascular Treatment for Small Core and Anterior Circulation Proximal Occlusion with Emphasis on Minimizing Computed Tomography (CT) to Recanalization Times" (ESCAPE) trial, 316 participants were enrolled at 22 centers worldwide ${ }^{7)}$. ESCAPE showed that the mechanical thrombectomy increased the rate of mRS ( 0 to 2 ) with favorable outcome (53.0\% vs. $29.3 \%$ in the control group; $p<0.001$ ). In case of proximal vessel occlusions with a small infarct core and moderate-to-good collaterals, rapid mechanical thrombectomy improved the functional outcome with reduced mortality.

The "Extending the Time for Thrombolysis in Emergency Neurological Deficits-Intra-Arterial” (EXEND-IA) enrolled
100 patients at 14 centers in Australia and New Zealand ${ }^{5)}$. Mechanical thrombectomy, which was performed at a median of 210 minutes after the onset of stroke, improved the mRS score (0 to 2 ) in 90 days.

The "Solitaire FR With the Intention for Thrombectomy as Primary Endovascular Treatment of Acute Ischemic Stroke" (SWIFT PRIME) enrolled 196 patients at 39 centers and analyzed cases of mechanical thrombectomy using a stent retriever within 6 hours of symptom onset ${ }^{14)}$. A favorable functional outcome (mRS 0 to 2 ) was observed in the mechanical thrombectomy group (60\% vs. $35 \%$ in the control group; $p<0.001$ ).

The "Endovascular Revascularization With Solitaire Device Versus Best Medical Therapy in Anterior Circulation Stroke Within 8 Hours" (REVASCAT) compared the functional outcomes between the mechanical thrombectomy group using the Solitaire stent retriever and the group undergoing only medical therapy. Mechanical thrombectomy performed within 8 hours of symptom onset increased functional independence (mRS 0 to 2 ) rate in 90 days ( $43.7 \%$ vs. $28.2 \%$ in the control group; adjusted odds ratio, 2.1; 95\% confidence interval, 1.1 to 4.0$)^{9)}$.

These five trials ${ }^{2,5,7,9,14)}$ published in 2015 consistently demonstrated that the emergent mechanical thrombectomy saved the penumbra of the acute hypo-perfused lesion, with a mortality rate similar to that of IV tPA therapy (Table 1). Among these trials, ESCAPE, EXTEND-IA, and SWIFT PRIME were terminated early because of favorable results in the endovascular thrombectomy group.

The Highly Effective Reperfusion evaluated in Multiple Endovascular Stroke Trials (HERMES) collaboration was based on a meta-analysis of the data from the five RCTs ${ }^{2,5,7,9,14)}$. The HERMES study was designed according to patient-level metaanalyses, which were more informative than the study-level meta-analyses. The HERMES study demonstrated the benefit of mechanical thrombectomy in most patients with acute ischemic stroke accompanied by proximal anterior circulation occlusion, irrespective of the patient characteristics or geographical location $^{8)}$.

The following two studies elicited the benefits of IV thrombolysis combined with mechanical thrombectomy. The study titled "Mechanical Thrombectomy after Intravenous Alteplase versus Alteplase Alone after Stroke” (THRACE) and published in 2016 demonstrated that mechanical thrombectomy combined with regular IV thrombolysis facilitated the treat- 
A Review of Intra-Arterial Thrombectomy | Sheen JJ, et al.

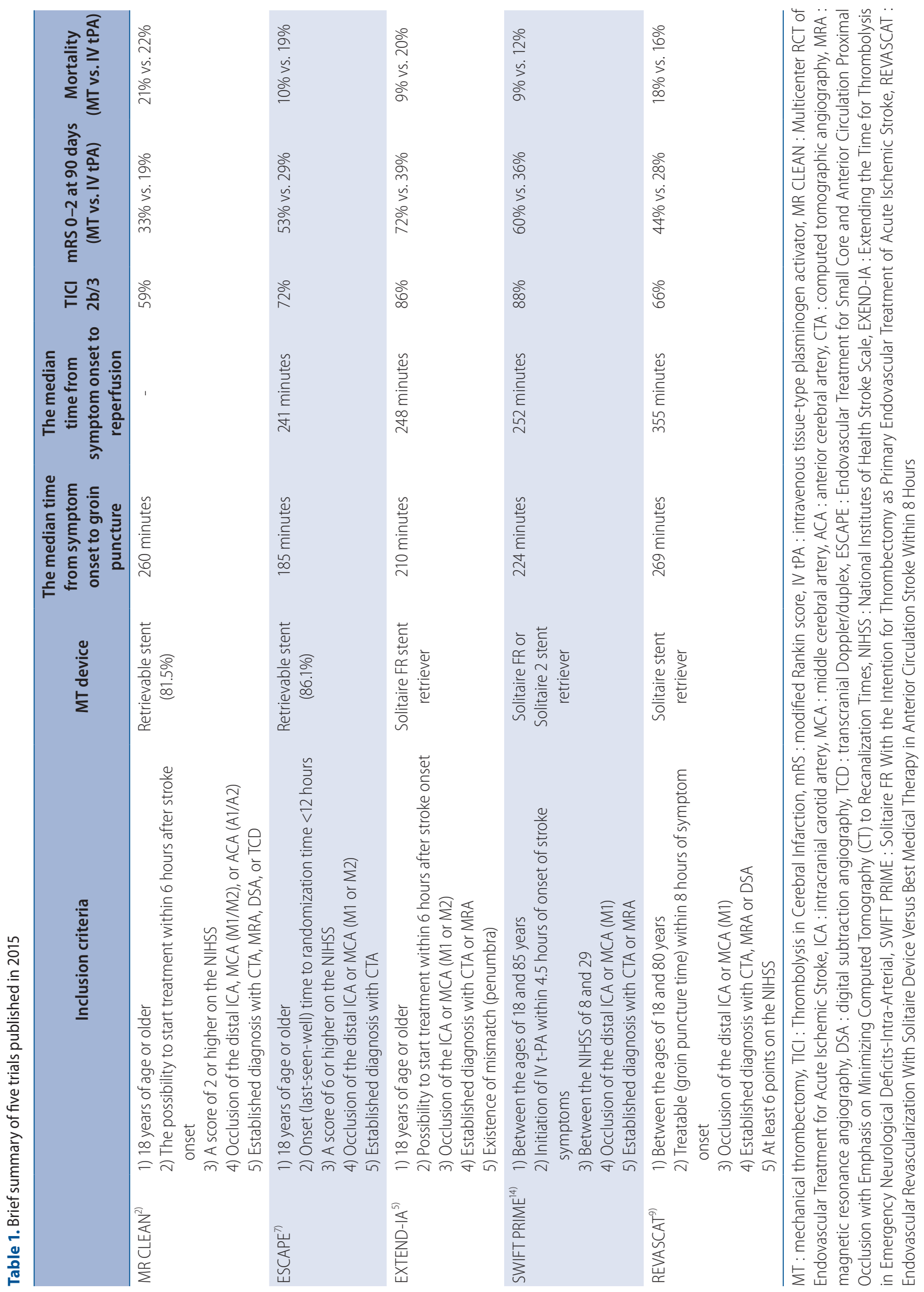


ment of patients with acute ischemic stroke ${ }^{3)}$. The study titled "Endovascular Therapy for Acute Ischemic Stroke: The Pragmatic Ischemic Stroke Thrombectomy Evaluation" (PISTE) also demonstrated that endovascular intervention was comparable to mechanical thrombectomy after CT angiography for rapid detection of large artery occlusion using IV alteplase and yielded excellent clinical outcomes with safety ${ }^{11}$.

\section{WHY DID THE PARADIGM SHIFT OCCUR?}

There are four reasons why the trials conducted in 2013 and 2015 showed different results ${ }^{13)}$. First, the three RCTs in 2013 used low-efficient thrombectomy devices ${ }^{8}$. These earlier studies were performed using intra-arterial fibrinolysis or primitive mechanical thrombectomy devices. The primitive mechanical devices such as Penumbra devices (Penumbra, CA, USA) and Mechanical Embolus Removal in Cerebral Ischemia (MERCI; Stryker, Kalamazoo, MI, USA) were used in the three RCTs published in 2013. On the other hand, the five successful RCTs were conducted using new therapeutic retrievable stents, such as Solitaire FR (Medtronic, Minneapolis, MN, USA). Newer-generation devices demonstrated good treatment outcomes because of higher recanalization rates and lower complication rates. Second, the three RCTs used insufficient image selection criteria $^{8)}$. In fact, the SYNTHESIS Expansion and IMS III did not require vascular imaging for patient selection. However, the five successful RCTs identified patients using vascular imaging and determined the salvageable tissue with a target-mismatch penumbral profile. They also performed an image test to determine collateral supply. Third, the three RCTs had a long door-to-recanalization time or did not report the time-to-reperfusion ${ }^{8}$. SYNTHESIS Expansion showed that the time interval from median onset to start of treatment in the mechanical thrombectomy group was 225 minutes (range, 194-260). In the IMS III study, the groin puncture time was $208 \pm 47$ minutes (mean \pm standard deviation) after stroke onset in the mechanical thrombectomy group. The onset-to-groin puncture time in the mechanical thrombectomy group in MR RESCUE was 381 74 minutes. Time-to-reperfusion was not reported in SYNTHESIS Expansion and MR RESCUE, but was 325 \pm 52 minutes in IMS III. Conversely, the five RCTs showed that the groin puncture time ranged from 51-269 minutes. The time from onset to re- perfusion ranged from 241-252 minutes, except in REVASCAT, which lasted 355 minutes. The results of time-to-reperfusion in the RCTs reported in 2015 were superior to those published in 2013. Fourth, there was low rate of successful recanalization in the three RCTs. SYNTHESIS did not demonstrate recanalization, while IMS III showed that the rate of recanalization for "Thrombolysis in Cerebral Infarction" (TICI) score of 2b-3 was 41\%. In MR RESCUE, there was 27\% revascularization (TICI score, 2 a to 3 ) in the mechanical embolectomy group. The rates of successful recanalization in MR CLEAN, ESCAPE, EXTEND-IA, SWIFT PRIME, and REVASCAT were 59\%, 72\%, 86\%, 88\%, and 66\%, respectively.

As a result, the five landmark studies in 2015 influenced the 2015 American Heart Association/American Stroke Association (AHA/ASA) guideline ${ }^{13)}$. In other words, the evidence collected in these five studies made mechanical thrombectomy the standard treatment for large vessel occlusion. The 2015 AHA/ASA guideline recommended that patients should undergo mechanical thrombectomy if they meet all the following criteria (level of evidence, A) : 1) pre-stroke mRS score 0 to $1 ; 2$ ) acute ischemic stroke receiving IV r-tPA within 4.5 hours of onset according to guidelines recommended by professional medical societies; 3 ) causative occlusion of the internal carotid artery or proximal middle cerebral artery (M1); 4) age $\geq 18$ years; 5) National Institutes of Health Stroke Scale (NIHSS) score of $\geq 6$; 6) Alberta stroke program early CT score of $\geq 6$; and 7) treatment initiated (groin puncture) within 6 hours of symptom onset ${ }^{13)}$. Since 2015, endovascular mechanical thrombectomy with IV-tPA has been the standard treatment for acute ischemic stroke caused by large vessel occlusions.

Recent studies have led to the extension of the mechanical thrombectomy time window. Time is the most important factor in the treatment of acute ischemic stroke. Current trends utilize advanced imaging protocols, such as diffusion-weighted imaging (DWI) and multi-channel CT perfusion (CTP), to efficiently identify core infarction, penumbra, and collateral flows. These efforts have shown that patient selection can overcome the barriers of time in specific cases (Fig. 1). In the "DWI or CTP Assessment with Clinical Mismatch in the Triage of Wake-Up and Late Presenting Strokes Undergoing Neurointervention with Trevo" (DAWN) trial, mechanical thrombectomy with standard medical treatment was compared to standard medical care alone for treatment of acute stroke patients who were considered to be well 6-24 hours 


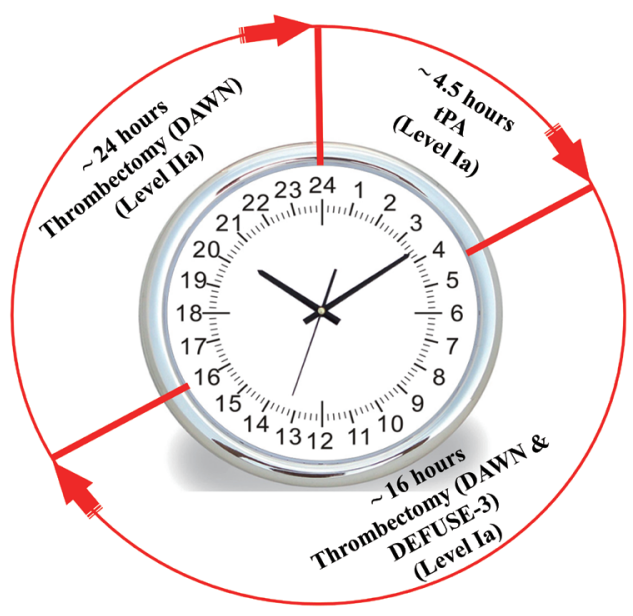

Fig. 1. 2018 Stroke guidelines extend the thrombectomy window to 24 hours. In selected patients with acute ischemic stroke who have large vessel occlusion in the anterior circulation, mechanical thrombectomy is recommended 1) within 6 to 16 hours of last known normal and meet DAWN or DEFUSE 3 eligibility criteria and 2) within 16 to 24 hours of last known normal and meet DAWN eligibility criteria. DAWN : DWI or CTP Assessment with Clinical Mismatch in the Triage of Wake-Up and Late Presenting Strokes Undergoing Neurointervention with Trevo, DEFUSE-3 : Endovascular Therapy Following Imaging Evaluation for Ischemic Stroke.

earlier, and had a penumbra related to mismatch between clinical deficit and infarct core. The DAWN trial defined the mismatch group according to the following imaging criteria : age $\geq 80$ years, NIHSS score $\geq 10$, and infarct core of $<21 \mathrm{~mL}$ for patients in group A; age $<80$ years, NIHSS score $\geq 10$, and infarct core of $<31 \mathrm{~mL}$ for patients in group $\mathrm{B}$; and age $<80$ years, NIHSS score $\geq 20$, and infarct core of $31-51 \mathrm{~mL}$ for patients in group $\mathrm{C}^{12}$. The DAWN trial showed that the mean score on the utility-weighted mRS at 90 days was 5.5 in the endovascular treatment group vs. 3.4 in the group receiving medical care alone; whereas, the rate of functional independence at 90 days was $49 \%$ in the former vs. $13 \%$ in the latter ${ }^{12)}$. These results showed no evidence of the effect of the treatment heterogeneity in the three subgroups. The "Endovascular Therapy Following Imaging Evaluation for Ischemic Stroke" (DEFUSE 3) trial enrolled patients who had penumbra in the brain area based on perfusion imaging, and those who arrived at the hospital 6-16 hours after they were last known to have been well. The important neuroimaging inclusion criterion of DEFUSE 3 was the mismatched profile on CT perfusion or magnetic resonance imaging (ischemic core volume $<70 \mathrm{~mL}$, mismatch ratio $>1.8$ and mismatch volume $>15 \mathrm{~mL})^{1)}$.
The DEFUSE 3 trial compared the endovascular mechanical thrombectomy group with the group receiving only standard medical treatment, wherein endovascular mechanical thrombectomy resulted in better functional outcomes ${ }^{1}$.

\section{CONCLUSION}

Currently, mechanical thrombectomy is the gold standard treatment for large artery occlusion with sufficient penumbra and small infarction core. The combined treatment of IV thrombolysis and mechanical thrombectomy should be performed rapidly in the early time window (within 6 hours), and careful patient selection based on imaging and clinical presentation is important in late time window (6-24 hours). Further studies are needed to show how much the early time window can be extended and provide effective diagnostic methods for patient selection in the late time window. Moreover, previous studies have focused on occlusive lesions of the anterior circulation, and further evidence will be needed for the treatment of occlusive lesions in the posterior circulation.

\section{CONFLICTS OF INTEREST}

No potential conflict of interest relevant to this article was reported.

\section{INFORMED CONSENT}

This type of study does not require informed consent.

\section{AUTHOR CONTRIBUTIONS}

\author{
Conceptualization : JJS, YWK \\ Data curation : JJS, YWK \\ Formal analysis : JJS, YWK \\ Funding acquisition : JJS, YWK \\ Methodology : JJS, YWK \\ Project administration : JJS, YWK \\ Visualization : JJS, YWK \\ Writing - original draft : JJS, YWK
}


Writing - review \& editing: JJS, YWK

\section{ORCID}

Jae Jon Sheen https://orcid.org/0000-0002-0474-5830

Young Woo Kim

\section{References}

1. Albers GW, Marks MP, Kemp S, Christensen S, Tsai JP, Ortega-Gutierrez $S$, et al. : Thrombectomy for stroke at 6 to 16 hours with selection by perfusion imaging. N Engl J Med 378 : 708-718, 2018

2. Berkhemer OA, Fransen PS, Beumer D, van den Berg LA, Lingsma HF, Yoo AJ, et al. : A randomized trial of intraarterial treatment for acute ischemic stroke. N Engl J Med 372 : 11-20, 2015

3. Bracard S, Ducrocq X, Mas JL, Soudant M, Oppenheim C, Moulin T, et al. : Mechanical thrombectomy after intravenous alteplase versus alteplase alone after stroke (THRACE): a randomised controlled trial. Lancet Neurol 15 : 1138-1147, 2016

4. Broderick JP, Palesch YY, Demchuk AM, Yeatts SD, Khatri P, Hill MD, et al. : Endovascular therapy after intravenous t-PA versus t-PA alone for stroke. N Engl J Med 368 : 893-903, 2013

5. Campbell BC, Mitchell PJ, Kleinig TJ, Dewey HM, Churilov L, Yassi N, et al. : Endovascular therapy for ischemic stroke with perfusion-imaging selection. N Engl J Med 372 : 1009-1018, 2015

6. Ciccone A, Valvassori L, Nichelatti M, Sgoifo A, Ponzio M, Sterzi R, et al. : Endovascular treatment for acute ischemic stroke. N Engl J Med 368 :

904-913, 2013

7. Goyal M, Demchuk AM, Menon BK, Eesa M, Rempel JL, Thornton J, et al. : Randomized assessment of rapid endovascular treatment of ischemic stroke. N Engl J Med 372 : 1019-1030, 2015

8. Goyal M, Menon BK, van Zwam WH, Dippel DW, Mitchell PJ, Demchuk AM, et al. : Endovascular thrombectomy after large-vessel ischaemic stroke: a meta-analysis of individual patient data from five randomised trials. Lancet 387 : 1723-1731, 2016

9. Jovin TG, Chamorro A, Cobo E, de Miquel MA, Molina CA, Rovira A, et al. : Thrombectomy within 8 hours after symptom onset in ischemic stroke. N Engl J Med 372 : 2296-2306, 2015

10. Kidwell CS, Jahan R, Gornbein J, Alger JR, Nenov V, Ajani Z, et al. : A trial of imaging selection and endovascular treatment for ischemic stroke. N Engl J Med 368 : 914-923, 2013

11. Muir KW, Ford GA, Messow CM, Ford I, Murray A, Clifton A, et al. : Endovascular therapy for acute ischaemic stroke: the Pragmatic Ischaemic Stroke Thrombectomy Evaluation (PISTE) randomised, controlled trial. J Neurol Neurosurg Psychiatry 88 : 38-44, 2017

12. Nogueira RG, Jadhav AP, Haussen DC, Bonafe A, Budzik RF, Bhuva P, et al. : Thrombectomy 6 to 24 hours after stroke with a mismatch between deficit and infarct. N Engl J Med 378 : 11-21, 2018

13. Powers WJ, Derdeyn CP, Biller J, Coffey CS, Hoh BL, Jauch EC, et al. : 2015 American Heart Association/American Stroke Association focused update of the 2013 guidelines for the early management of patients with acute ischemic stroke regarding endovascular treatment: a guideline for healthcare professionals from the American Heart Association/American Stroke Association. Stroke 46 : 3020-3035, 2015

14. Saver JL, Goyal M, Bonafe A, Diener HC, Levy El, Pereira VM, et al. : Stent-retriever thrombectomy after intravenous t-PA vs. t-PA alone in stroke. N Engl J Med 372 : 2285-2295, 2015 